\title{
Pulsed Antennas
}

\author{
GIORGIO FRANCESCHETTI, SENIOR MEMBer, IEEe, AND CHARLES H. PAPAS, MEMber, IEeE
}

\begin{abstract}
The problem of pulsed antennas has two complementary parts: i) analysis of the radiation field when the driving voltage is given and ii) synthesis of the driven voltage when the radiation field is given. In this paper a number of heuristic procedures are presented, relating to the computation of transient radiation from elementary sources, coaxial apertures, infinitely long cylindrical antennas, finite cylindrical antennas, and loop antennas. Comparison with ayailable rigorous solutions and experiments is also provided.
\end{abstract}

\section{INTRODUCTION AND DISCUSSION OF PRINCIPAL RESULTS}

In early 1969, Prof. T. T. Wu stated: "It is worth emphasizing that our knowledge about the transient response of antennas is very meager indeed. Any progress in this rather neglected field is certainly going to be of tremendous value" [1]. Although a few new papers have appeared on this subject since then, the above statement is probably still valid.

Most of the papers concerned with transient currents on and transient radiation from antennas use solutions of frequency-domain integral equations which are then transformed into the time-domain by Fourier techniques. These papers cover the transient behavior of the infinite cylindrical antenna [2] [7], the finite cylindrical antenna driven by a coaxial line [8], or by a gap type excitation $[9],[10]$, the conical antenna [11], the aperture antenna $[12]-[14]$, the loop antenna [15], [16], and the loaded antenna [17]. In a few papers, an experimental verification of the theory is provided, with reference to the radiated and received fields [18], [19] or to the input response of the antennas [20], [21]. Also, the effects of thin-wire approximations and of source-excitation modeling can be found in the literature [31], [32]. Other approaches that have been used are the singularity expansion method [22], [23], the solution of the transient current integral equation directly in time-domain [24]-[29], and an almost rigorous application of the Wiener-Hopf technique [30].

A general characteristic of most of the referenced material is the emphasis on either the extensive use of numerical methods or the presentation of the solution in an integral form that must be evaluated numerically or at least asymptotically [7]. A somewhat different approach has been suggested [33] which aims at the general properties (if any) of transient radiation. In this paper, this approach is formalized and further developed.

Manuscript received December 20, 1973; revised April 8, 1974. G. Franceschetti was with the California Institute of Technology, Pasadena, Calif., on leave from the Department of Electrical Engineering, University of Naples, Naples, Italy.

C. H. Papas is with the California Institute of Technology, Pasadena, Calif. 91109.
In Section II, the transient radiation from elementary electric or magnetic sources with prescribed current distributions is analyzed by using a formal Fourier inversion of known steady-state results. The source environment is assumed not only isotropic and homogeneous, but also nondispersive, since completely different results are obtained when dispersion is taken into account [34]-[37]. This assumption will apply throughout this paper. The analysis shows that the transient radiated power is expressed in terms of the $1 / r$ components of the field only, when the antenna excitation is confined in time. These components in the case of an electric dipole are proportional to the time-derivative of the current $\dot{I}\left(t^{*}\right)$ and to the second time-derivative of the input voltage $\ddot{V}\left(t^{*}\right)$, at the retarded time $t^{*}=t-r / c$. In the case of a magnetic dipole, the $1 / r$ fields are proportional to $\ddot{I}\left(t^{*}\right)$, $\dot{V}\left(t^{*}\right)$, where $I(t), V(t)$ are, respectively, the current along, and the voltage across the small current loop.

In Section III, a deeper analysis of transient radiation from the elementary sources is presented. For the electric dipole, a model of two opposite charges, which collapse one upon the other, is assumed. For the magnetic dipole, a pulsed current along a circular loop is considered. In each case, it is shown that the far-field waveform is not proportional to the first or second time derivatives of $V(r)$ or $I(t)$, when the time resolution is greater than $h / c, h$ being a characteristic linear dimension of the antenna, and $c$ the light velocity. In this same scale, the radiation due to an assumed pulse of current appears in the form of two pulses (electric dipole), or a continuous radiated waveform with square-root singularities (magnetic dipole). However, when a time scale larger than $h / c$ is considered, it is shown that a proper limiting process leads to the formal results of Section II. From a physical standpoint, the process can be understood as a contraction of the physical dimensions of the radiators, or equivalently, as a limited time-resolution of the field measuring devices.

Also the more complex radiating structures that will be considered here are subject to the above time-resolution property. Accordingly, a macroscopic and a microscopic approach are defined for the transient radiation, with respect to a time-resolution of order $h / c$. In all cases the transition from one time-scale to the other is developed in detail.

In Section IV, an analysis of transient radiation from coaxial apertures is developed. In the macroscopic approach, advantage is taken of the equivalence between the aperture field and a distributed magnetic loop [38]. The far-field is shown to be proportional to $\ddot{V}\left(t^{*}\right), V(t)$ being the voltage across the aperture. In the microscopic ap- 
proach, the far-field is computed as due to the sudden reflection of the current at the open end along the edges of the conductors of the coaxial aperture. The radiated waveform is characterized by four square-root singularities, where the incident voltage is a pulse and the coaxial aperture is circular. These results are in agreement with a quasi-rigorous solution of the problem [30].

In Section $V$ the radiation coming from the gap of a linear antenna is considered. In the macroscopic approach, the far-field turns out to be proportional to $\dot{V}\left(t^{*}\right)$. In the microscopic approach, the radiated waveform has a squareroot singularity when the applied voltage is a step. This last result is consistent with the early-time behavior of the far-field radiated by an infinitely long cylindrical antenna [7].

In Section VI, an approximate method is developed for computing transient radiation from linear antennas and loops large with respect to $c T, T$ being the pulse width. The approximation involved is the use of transmission line analysis for modeling currents and voltages. This results in a sinusoidal current distribution. It is therefore expected that the results of the analysis are valid in the limit of vanishingly small wire radius [39].

For the linear antenna, the far-field turns out to be radiated from the input terminals and the end-tips, as qualitatively suggested in previous analyses [11], [13] and experimentally confirmed [18]. For the loop antenna, the far-field appears in the form of a continuous waveform, with square-root singularities (when the applied voltage is a Dirac pulse). For each observational position there are two flash points on the loop from which most of the radiation appears to be emanating.

In Section VII, conclusions and recommendations are made. The suggested recipes and techniques of analysis are simple and rather general and allow the handling of a large variety of radiators of practical interest. However, the dispersion of the pulsed waveforms propagating along the radiating structures is not taken into account. On the other hand, this dispersion will produce a continuous radiation, which is generally small and gives the late-time response of the radiating structure [7].

\section{FORMAL SOLUTION FOR TRANSIENT RADIATION FROM ELEMENTARY SOURCES}

Suppose we have an infinitesimal oscillating electric dipole of moment $p_{e}=p_{0} \exp [-i \omega t]$ in a homogeneous isotropic environment. The dipole is located at the origin of a spherical coordinate system $(r, \theta, \phi)$ and is oriented parallel to the direction $\theta=0$. The components of the dipole's electromagnetic transformed field are given by the well-known expressions

$$
\begin{aligned}
& \widehat{E}_{r}(\omega)=\zeta \frac{2 p_{0} \exp [i \omega r / c]}{4 \pi r} \cos \theta\left[-\frac{i \omega}{r}+\frac{c}{r^{2}}\right] \\
& \widehat{E}_{\theta}(\omega)=\zeta \frac{p_{0} \exp [i \omega r / c]}{4 \pi r} \sin \theta\left[\frac{(i \omega)^{2}}{c}-\frac{i \omega}{r}+\frac{c}{r^{2}}\right]
\end{aligned}
$$

$$
\widehat{H}_{\phi}(\omega)=\frac{p_{0} \exp [i \omega r / c]}{4 \pi r^{r}} \sin \theta\left[\frac{(i \omega)^{2}}{c}-\frac{i \omega}{r}\right]
$$

where $\zeta=(\mu / \epsilon)^{1 / 2}$ and $c=1 /(\epsilon \mu)^{1 / 2}$.

From (1) the transient fields can be easily obtained for the case of a nondispersive environment, when the dipole moment varies arbitrarily in time, i.e., $p_{e}=p_{e}(t)$. By taking the Fourier transform of (1)

$$
E_{r}(t)=\frac{1}{2 \pi} \int_{-\infty}^{+\infty} \hat{E}_{r}(\omega) \exp [-i \omega t] d \omega
$$

and doing the same for the other field components, it is easily obtained

$$
\begin{aligned}
& E_{r}(t)=\frac{2 \zeta}{4 \pi r} \cos \theta\left[\frac{\dot{p}_{e}\left(t^{*}\right)}{r}+\frac{c p_{e}\left(t^{*}\right)}{r^{2}}\right] \\
& E_{\theta}(t)=\frac{\zeta}{4 \pi r} \sin \theta\left[\frac{\ddot{p}_{e}\left(t^{*}\right)}{c}+\frac{\dot{p}_{e}\left(t^{*}\right)}{r}+\frac{c p_{e}\left(t^{*}\right)}{r^{2}}\right] \\
& H_{\phi}(t)=\frac{1}{4 \pi r} \sin \theta\left[\frac{\ddot{p}_{e}\left(t^{*}\right)}{c}+\frac{\dot{p}_{e}\left(t^{*}\right)}{r}\right]
\end{aligned}
$$

where $t^{*}=t-r / c$ is the retarded time, and a dot means derivation with respect to time.

The corresponding expressions for the magnetic dipole are immediately obtained by duality $(H \rightarrow E, E \rightarrow-H$, $\left.\zeta \rightarrow 1 / \zeta, p_{e} \rightarrow-p_{m}\right)$. Thus

$$
\begin{aligned}
& H_{r}(t)=\frac{2}{4 \pi r \zeta} \cos \theta\left[\frac{\dot{p}_{m}\left(t^{*}\right)}{r}+\frac{c p_{m}\left(t^{*}\right)}{r^{2}}\right] \\
& H_{\theta}(t)=\frac{1}{4 \pi r \zeta} \sin \theta\left[\frac{\ddot{p}_{m}\left(t^{*}\right)}{c}+\frac{\dot{p}_{m}\left(t^{*}\right)}{r}+\frac{c p_{m}\left(t^{*}\right)}{r^{2}}\right] \\
& E_{\phi}(t)=-\frac{1}{4 \pi r} \sin \theta\left[\frac{\ddot{p}_{m}\left(t^{*}\right)}{c}+\frac{\dot{p}_{m}\left(t^{*}\right)}{r}\right] .
\end{aligned}
$$

The r-component of the Poynting vector is

$$
S_{r}{ }^{e}(t)=E_{\theta}(t) H_{\phi}(t) \quad S_{r}{ }^{m}(t)=-E_{\phi}(t) H_{\theta}(t)
$$

for the electric and the magnetic dipole, respectively. The radiated energy density at $(r, \theta)$ is

$$
\begin{aligned}
w^{e}(r, \theta)= & \int_{-\infty}^{+\infty} S_{r}^{e}(t) d t=\frac{\zeta \sin ^{2} \theta}{(4 \pi r)^{2} c^{2}}\left\{\int_{-\infty}^{+\infty} \ddot{p}_{e}^{2} d t\right. \\
& \left.+\frac{c}{r}\left[\dot{p}_{e}{ }^{2}+\left(\frac{c}{r}\right) \dot{p}_{e} p_{e}+\frac{1}{2}\left(\frac{c}{r}\right)^{2} p_{e}{ }^{2}\right]_{-\infty}^{+\infty}\right\} \\
w^{m}(r, \theta)= & \int_{-\infty}^{+\infty} S_{\mathrm{r}}{ }^{m}(t) d t=\frac{1}{\zeta} \frac{\sin ^{2} \theta}{(4 \pi r)^{2} c^{2}}\left\{\int_{-\infty}^{+\infty} \ddot{p}_{m} d t\right. \\
& \left.+\frac{c}{r}\left[\dot{p}_{m}^{2}+\frac{c}{r} \dot{p}_{m} p_{m}+\frac{1}{2}\left(\frac{c}{r}\right)^{2} p_{m^{2}}\right]_{-\infty}^{+\infty}\right\}
\end{aligned}
$$

When the dipole excitation has a finite duration the bracketed terms inside the large parentheses are equal to 
zero, and (6) transform as

$$
\begin{aligned}
w^{e}(r, \theta) & =\frac{\zeta \sin ^{2} \theta}{(4 \pi r)^{2} c^{2}} \int_{-\infty}^{+\infty} \ddot{p}_{e}(t) d t \\
w^{m}(r, \theta) & =\frac{\sin ^{2} \theta}{\zeta(4 \pi r)^{2} c^{2}} \int_{-\infty}^{+\infty} \ddot{p}_{m}(t) d t .
\end{aligned}
$$

Equations (7) show that the total radiated energy is dependent only on the $1 / r$ components of the field accordingly identified as the "radiative" terms.

It is interesting to calculate in which regions of space the radiative terms are the dominant field components. Reference is made to a Gaussian pulse of electric moment

$$
p_{e}(t)=p_{0} \exp \left[\frac{-2 t^{2}}{T^{2}}\right]
$$

$T$ being the effective width of the pulse (distance between inflection points). Substituting (8) in (3) one obtains

$$
\begin{aligned}
E_{r}(t)= & \frac{2 \zeta p_{e}\left(t^{*}\right)}{4 \pi r(c T)^{2}} \cos \theta\left[-4 \frac{c T}{r} \frac{t^{*}}{T}+\left(\frac{c T}{r}\right)^{2}\right] \\
E_{\theta}(t)= & \frac{\zeta p_{e}\left(t^{*}\right)}{4 \pi r(c T)^{2}} \\
& \cdot \sin \theta\left[\left\{8\left(\frac{t^{*}}{T}\right)-4\right\}-4 \frac{c T}{r} \frac{t^{*}}{T}+\left(\frac{c T}{r}\right)^{2}\right] \\
H_{\phi}(t)= & \frac{p_{e}\left(t^{*}\right)}{4 \pi r(c T)^{2}} \sin \theta\left[\left\{8\left(\frac{t^{*}}{T}\right)-4\right\}-4 \frac{c T}{r} \frac{t^{*}}{T}\right] .
\end{aligned}
$$

It follows from (9) that the radiative terms are always dominant provided that

$$
r \gg c T
$$

save in the neighborhood of $t^{*}=T / \sqrt{2}$, where the radiative terms are zero. Identical results are obtained for the case of a magnetic dipole. It is concluded that the spherical surface $r=c T$ divides the space into two regions, which can be labeled as inductive space (inner region) and radiative space (outer region). In the following, only radiative fields will be considered.

Equations (3) and (4) can be used for computing radiation from "small" dipoles and loops, where "small" applies to the $c T$ space scale. The following results are obtained:

i) Electric short dipole of effective height $h$ :

$$
\begin{aligned}
& E_{\theta}(t)=\zeta H_{\phi}(t) \\
& H_{\phi}(t)=-\frac{\dot{I}\left(t^{*}\right) h}{4 \pi r c} \sin \theta=-\frac{C h \ddot{V}\left(t^{*}\right)}{4 \pi r c} \sin \theta
\end{aligned}
$$

ii) Small loop of area $A$ :

$$
\begin{aligned}
& H_{\theta}(t)=\frac{\ddot{I}\left(t^{*}\right) A}{4 \pi r c^{2}} \sin \theta=-\frac{\dot{V}\left(t^{*}\right) A}{4 \pi r c^{2} L} \sin \theta \\
& E_{\phi}(t)=-\zeta H_{\theta}(t)
\end{aligned}
$$
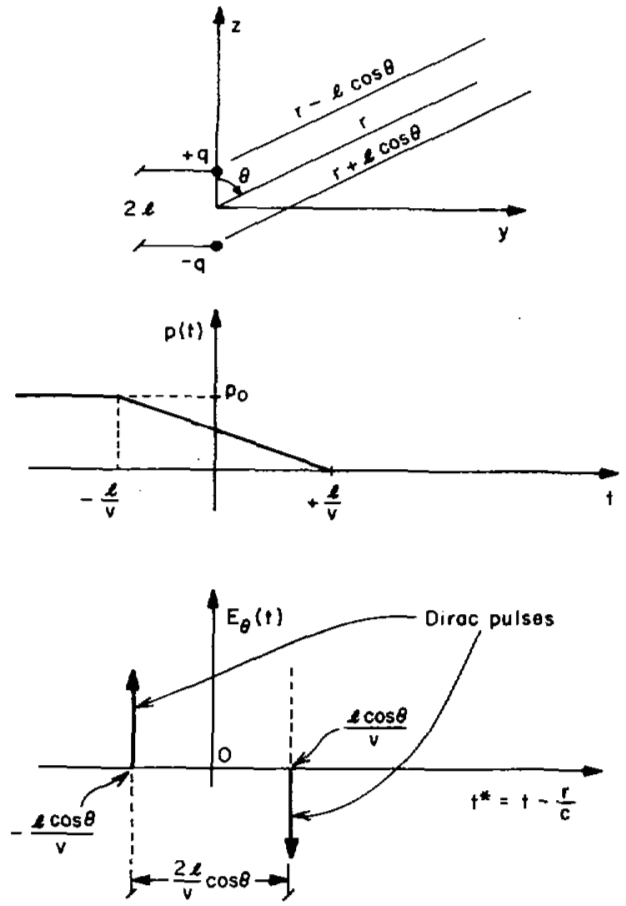
Fig. 1. Relevant to physical model for computing transient radia-

In (10) and (11) $C$ and $L$ are the static capacitance and inductance of the dipole and the loop, respectively, $I(t)$ and $V(t)$ the current along and the voltage across the radiators, and the quasi-static relations

$$
\text { (dipole) } I(t)=C \dot{V}(t) \quad(\text { loop }) V(t)=-L \dot{I}(t)
$$

have been used.

Equations (10) and (11) clearly show that the radiative fields are not a faithful replica of the transmitted signals (currents or voltages on the radiators). In particular, if the current is proportional to a Dirac pulse $\delta(t)$ (electric dipole), and to a step function $U(t)$ (magnetic dipole), the radiative field turns out to be proportional to $\ddot{\delta}(t)$. Therefore, the radiative field flip-flops [33].

\section{PHYSICS OF TRANSIENT RADIATION FROM ELENENTARY SOURCES}

In this section a deeper analysis of transient radiation from elementary sources will be made.

For the case of an electric dipole, reference is made to Fig. 1. Two charges, $+q$ and $-q$, are located at a distance $2 l$ apart. At a time $t=-l / v$ the charge $+q$ is suddenly accelerated and is then made to travel at constant velocity $v$, so that it collapses on the charge $-q$ at time $t=l / v$. The plot of the electric moment $p(t)$ of the system versus time is given in the graph of Fig. 1. Accordingly, a pulsed current is flowing in the time interval $|t| \leq l / c$, from $z=l$ to $z=-l$. 
Formal espressions of current density in the time and frequency domains are the following:

$$
\begin{aligned}
J(r, t) & =-e_{z} q v \delta(x) \delta(y) \delta(z+v t) & & \\
& =-e_{z} q \delta(x) \delta(y) \delta\left(t+\frac{z}{v}\right), & & |t| \leq \frac{l}{v} \\
J(r, t) & =0, & & |t|>\frac{l}{v} \\
\hat{J}(r, \omega) & =-e_{z} q \delta(x) \delta(y) \exp \left[-i \omega \frac{z}{v}\right], & & |z| \leq l \\
\hat{J}(r, \omega) & =0, & & |z|>l
\end{aligned}
$$

$\boldsymbol{e}_{z}$ being a unit vector in the $z$ direction. The transformed radiative fields associated with (14) are

$$
\begin{gathered}
\widehat{E}_{\theta}(\omega)=-i \zeta \frac{\exp [i \omega r / c]}{2 \pi r} q \frac{\sin [(\omega l / c)(\eta+\cos \theta)]}{\eta+\cos \theta} \sin \theta \\
\widehat{H}_{\phi}(\omega)=\frac{\widehat{E}_{\theta}(\omega)}{\zeta} \quad \eta=\frac{c}{v} .
\end{gathered}
$$

By Fourier transforming (15) into the time-domain, the result

$$
\begin{aligned}
& E_{\theta}(t)= \zeta \frac{q}{4 \pi r} \frac{\sin \theta}{\eta+\cos \theta} \\
& \cdot\left[\delta\left(t^{*}+\frac{l}{v}+\frac{l}{c} \cos \theta\right)-\delta\left(t^{*}-\frac{l}{v}-\frac{l}{c} \cos \theta\right)\right] \\
& H_{\phi}(t)=\frac{E_{\theta}}{\zeta} \quad t^{*}=t-\frac{r}{c}
\end{aligned}
$$

is obtained. Equations (16) clearly show that the radiation emanates from the end points of the configuration at times $-l / v$ (acceleration) and $+l / v$ (deceleration), respectively (see the diagram of Fig. 1). Accordingly, the radiated field is not proportional to the time derivative of the current (see (10)), when a time scale less than $2 l / v$ is considered.

The transition from (16)-microscopic approach-to (10) - macroscopic approach-is readily accomplished by letting $2 l / v$ approach zero. One obtains

$$
\begin{aligned}
E_{\theta}(t) & =\lim _{2 l / t \rightarrow 0} \zeta \frac{2 q l}{4 \pi r c} \sin \theta \frac{\delta\left(t^{*}+x\right)-\delta\left(t^{*}-x\right)}{2 x} \\
& =\zeta \frac{p_{0}}{4 \pi r c} \sin \theta \dot{\delta}\left(t^{*}\right), \quad x=\frac{l}{c}(\eta+\cos \theta) \\
H_{\phi}(t) & =\zeta E_{\theta}
\end{aligned}
$$

thus recovering (10), since $I(t) 2 l=-q \delta(t) 2 l=-p_{0} \delta(t)$.

For the case of a magnetic dipole, reference is made to Fig. 2. A constant current $I_{0}$ is suddenly switched on at time $t=-\tau$ and switched off at time $t=+\tau$. The current

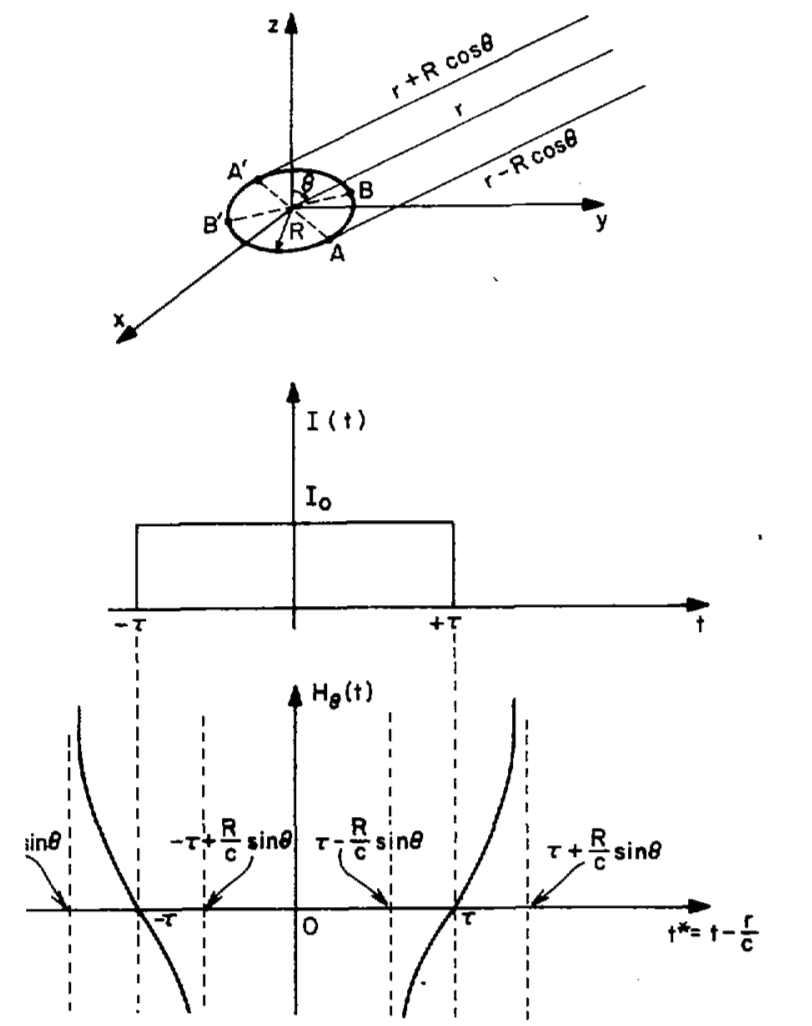

Fig. 2. Relevant to physical model for computing transient radiation from magnetic dipole .

flows in a circular loop of radius $R$ located in the plane $(x, y)$.

Formal expressions of the current in the time and frequency domains are the following:

$$
\begin{aligned}
I(t) & =I_{0}[U(t+\tau)-U(t-\tau)] \\
\hat{I}(\omega) & =2 I_{0} \frac{\sin \omega \tau}{\omega} .
\end{aligned}
$$

The transformed radiative fields associated with (19) are $\hat{H}_{\theta}(\omega)=-\frac{R I_{0}}{r c} \sin (\omega \tau) J_{1}\left(\frac{\omega}{c} R \sin \theta\right) \exp \left[i \frac{\omega}{c} r\right]$

$\widehat{E}_{\phi}(\omega)=-\zeta \hat{H}_{\theta}$

$J_{1}(x)$ being the Bessel function of first kind.

By Fourier transforming (20) into the time-domain, the result

$$
\begin{aligned}
H_{\theta}(t) & =-\frac{I_{0}}{2 \pi r \sin \theta}\left[H_{\theta}^{+}+H_{\theta}^{-}\right] \\
E_{\phi}(t) & =-\zeta H_{\theta} \\
H_{\theta} \pm(t) & = \pm \frac{t^{*} \pm \tau}{\left[((R / c) \sin \theta)^{2}-\left(t^{*} \pm \tau\right)^{2}\right]^{1 / 2}}, \\
& |t \pm \tau| \leq \frac{R}{c} \sin \theta
\end{aligned}
$$

$$
H_{\theta}^{ \pm}\left(t^{*}\right)=0, \quad|t \pm \tau|>\frac{R}{c} \sin \theta
$$

is obtained. 


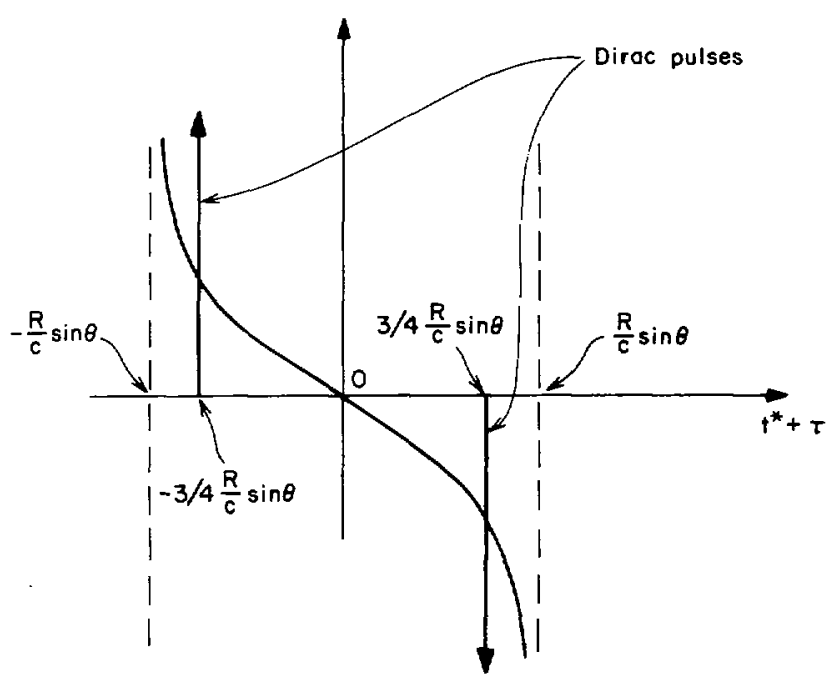

Fig. 3. Relevant to transition from continuous radiation to equivalent pulses.

Equations (22) clearly show that the antenna radiates at times $t=-\tau, t=\tau$. For each direction of observation there is continuous radiation for $|t-\tau| \leq R \sin \theta / c$, characterized by square-root singularies (see the diagram of Fig. 2). The radiation emanates mainly from points $A A^{\prime}$ (flash points) at the intersections of the loop with the plane containing the direction of observation and the $z$ axis. No radiation emanates from points $B B^{\prime}$ (black points), due to a cancellation effect. Accordingly, the radiated field is not proportional to the second time derivative of the current (see (11)), when a time scale less than $R / c$ is considered.

The transition from (21)-microscopic approach-to (11) - macroscopic approach - is accomplished by letting $R / c$ approach zero.

Reference is made to the radiation which takes place at $t=-\tau$. For $R \rightarrow 0$, it seems quite reasonable to substitute for the actual radiated waveform some equivalent pulses (see Fig. 3). Reasonable requirements are: i) the strength of each pulse must be equal to the time integral of the actual waveform and ii) the time allocation of each pulse must divide the actual waveform into parts of equal area. When these requirements are fulfilled, (21) and (22) transform into

$$
\begin{aligned}
H_{\theta}(t) & =\lim _{R / c \rightarrow 0} \frac{I_{0} R}{2 \pi r c} \frac{\left[\delta\left(t^{*}+\tau+\frac{3}{4} x\right)-\delta\left(t^{*}+\tau-\frac{3}{4} x\right)\right]}{x} \\
& =\frac{3}{\pi} \frac{I_{0} \pi R^{2}}{4 \pi r c^{2}} \sin \theta \dot{\delta}\left(t^{*}+\tau\right) \\
E_{\phi}(t) & =-\zeta H_{\theta}(t), \quad x=\frac{R}{c} \sin \theta
\end{aligned}
$$

thus recovering (11), since $I(t)=U(t+\tau)$, apart from the factor $3 / \pi=0.995 \simeq 1$ (depending upon the somewhat arbitrary identification between actual radiated waveforms and equivalent pulses).

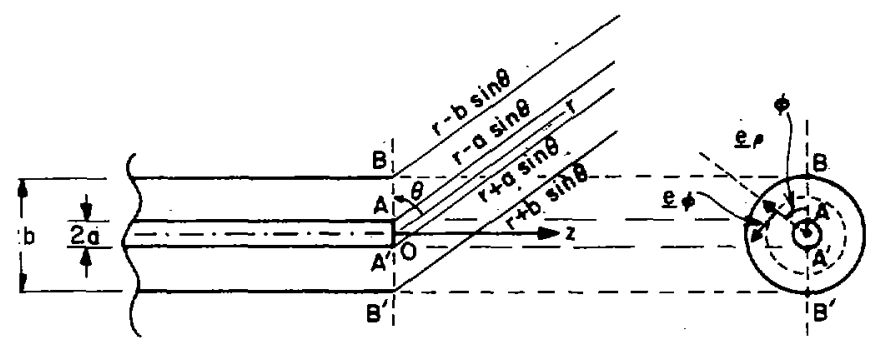

Fig. 4. Relevant to transient radiation from coaxial apertures.

\section{TRANSIENT RADIATION FROM COAXIAL APERTURES}

In this section, the transient radiation from coaxial apertures will be considered. Reference is made to Fig. 4 where the radiation from an open ended coaxial cable of circular cross section is considered. However, the results which will be presented can readily be generalized to apply to radiating apertures of different geometry.

First, the macroscopic approach will be exploited. Let $V(t)$ be the incident voltage at the open end of the coaxial cable. Under the assumption of perfect open-circuit conditions, the electric field in the aperture has only a $\rho-$ component given by

$$
E_{\rho}(t)=\frac{2 V(t)}{\rho \ln b / a}
$$

and the magnetic field is zero.

By using the equivalence theorem, the radiated field can be computed as due to an equivalent magnetic current density with only a component, $J_{m \phi}(t)=E_{\rho}(t)$. A $z$ directed electric dipole of moment given by [38]

$$
\begin{aligned}
p_{e}(t) & =\frac{2 V(t) \epsilon}{\ln b / a} \int_{a}^{b} \frac{1}{r} \pi r^{2} d r=\pi \epsilon \frac{V(t)}{\ln b / a}\left(b^{2}-a^{2}\right) \\
& =\frac{A}{2 \pi c Z_{0}} V(\dot{t})
\end{aligned}
$$

is therefore associated to the aperture. In (25) $A=$ $\pi\left(b^{2}-a^{2}\right)$ and is the aperture area, and $Z_{0}=[\zeta \ln b / a] / 2 \pi$ and is the characteristic impedance of the coaxial cable.

When $A$ is small compared to $(c T)^{2}$, the higher order moments associated with the aperture are negligible, and the radiated field can be obtained from the results of Sction II. Hence

$$
\begin{aligned}
E_{\theta}(t) & =\frac{\zeta}{Z_{0}} \frac{A}{2 \pi} \frac{\ddot{V}\left(t^{*}\right)}{3 \pi r c^{2}} \sin \theta \\
H_{\phi}(t) & =\frac{E_{\theta}(t)}{\zeta}
\end{aligned}
$$

Accordingly, the radiated field is proportional to the second time-derivative of the incident voltage.

As a preliminary to the study of the radiation from the microscopic point of view a model similar to that of Section II for the electric dipole is considered. A charge $q$ at 
position $z=-l$ is suddenly accelerated at time $t=-z / v$ and is then made to travel at constant velocity $v$ along the positive direction of the $z$ axis. At $t=0$ and $z=0$, the charge is suddenly reflected and is again made to travel at constant velocity $v$ along the negative direction of the $z$ axis. At $t=z / v$ and $z=-l$ the charge is suddenly stopped.

The radiated field can be computed by following the same procedure as in Section III. Only the results are given in the following. The field is radiated at times $t=-l / v, 0$, $l / v$, from points $z=-l, 0, l$, respectively. Particularly, the field radiated at $t=0, z=0$ (reflection of the charge) is given by

$$
\begin{aligned}
& E_{\theta}(t)=-\zeta \frac{q}{2 \pi r} \frac{\eta \sin \theta}{\eta^{2}-\cos ^{2} \theta} \delta\left(t^{*}\right) \\
& H_{\phi}(t)=\frac{E_{\theta}(t)}{\zeta}, \quad \eta=\frac{c}{v} .
\end{aligned}
$$

Turning to the coaxial structure of Fig. 4, let $I(t)=$ $q \delta(t-z / c)$ be the current propagating along the cable. The pulsed linear charge densities on the inner and outer conductor will be $q / 2 \pi a$ and $-q / 2 \pi b$, respectively. These pulsed charges will apparently be propagated with velocity $c$ along the cable. Accordingly, the radiated field can be computed via (27) (with $\eta=1$ ) and superposition. Hence

$$
\begin{aligned}
E_{\theta}(t)=\zeta & \frac{q}{(2 \pi)^{2} r \sin \theta} \\
\cdot & {\left[\int_{0}^{2 \pi} \delta\left(t-\frac{r_{b}}{c}\right) d \phi-\int_{0}^{2 \pi} \delta\left(t-\frac{r_{a}}{c}\right) d \phi\right] }
\end{aligned}
$$

where $r_{a, b}$ are the distances of the point $(r, \theta)$ from the inner and outer rims of the cable, respectively,

$$
r_{a} \simeq r-a \sin \theta \cos \phi \quad r_{b} \simeq r-b \sin \theta \cos \phi .
$$

In order to compute the integrals which appear in (29) it is convenient to use the Fourier transform

$$
\begin{aligned}
& \int_{-\infty}^{+\infty} d t \exp [i \omega t] \int_{0}^{2 \pi} \delta\left(t^{*}+\frac{b}{c} \sin \theta \cos \phi\right) d \phi \\
& =\int_{0}^{2 \pi} d \phi \int_{-\infty}^{+\infty} \delta\left(t^{*}+\frac{b}{c} \sin \theta \cos \phi\right) \exp [i \omega t] d t \\
& =\exp \left[i \frac{\omega}{c} r\right] \int_{0}^{2 \pi} d \phi \exp \left[-\frac{i \omega}{c} b \sin \theta \cos \phi\right] \\
& =2 \pi \exp \left[i \frac{\omega}{c} r\right] J_{0}\left(\frac{\omega}{c} b \sin \theta\right)
\end{aligned}
$$

and similarly for the other integral. By Fourier inverting (30) in the time-domain and substituting in (29), the result

$$
E_{\theta}(t)=\zeta \frac{2}{\pi} \frac{q}{4 \pi r \sin \theta}\left[E^{b}\left(t^{*}\right)-E^{a}\left(t^{*}\right)\right]
$$

$$
H_{\phi}(t)=\frac{E_{\theta}(t)}{\zeta}
$$

is obtained. In (31) the functions $E^{a, b}\left(l^{*}\right)$ are given by

$$
\begin{array}{ll}
E^{x}\left(t^{*}\right)=\frac{1}{\left[((x / c) \sin \theta)^{2}-t^{* 2}\right]^{1 / 2}}, & \left|t^{*}\right| \leq \frac{x}{c} \sin \theta \\
E^{x}\left(t^{*}\right)=0, & \left|t^{*}\right|>\frac{x}{c} \sin \theta .
\end{array}
$$

A plot of the radiated field is given in Fig. 5. The preceding results are in complete agreement with those of an exact analysis carried out by using Wiener-Hopf techniques [30] when pulses such that $c T \ll b$ are considered. This last restriction does not apply in the proximity of the forward direction.

Equations (31) and (32) clearly show that, for each direction of observation, there is a continuous radiation for $\left|t^{*}\right| \leq b \sin \theta / c$ characterized by square-root singularities. The radiation emanates mainly from points $A A^{\prime}, B B^{\prime}$ (flash points). Accordingly, the radiated field is not proportional to the second time-derivative of the incident voltage $V(t)=Z_{0} q \delta(t)=\bar{V} \delta(t)$ (see $\left.(26)\right)$, when a time scale less than $b / c$ is considered.

Also in this case the transition from (31)-microscopic approach-to (26)-macroscopic approach-can be accomplished by substituting for the actual radiated waveform equivalent pulses (see Fig. 5) as already done in Section III. Then $b / c$ and $a / c$ are required to approach zero. One obtains (see Fig. 5)

$$
\begin{aligned}
E_{\theta}(t)= & \lim _{b / c \rightarrow 0 ; a / c \rightarrow 0} \frac{\zeta}{Z_{0}} \frac{\bar{V}}{4 \pi r \sin \theta} \\
& \cdot\left[(x-y) \frac{\delta\left(t^{*}-x\right)-\delta\left(t^{*}-y\right)}{x-y}\right. \\
& \left.-(x-y) \frac{\delta\left(t^{*}+y\right)-\delta\left(t^{*}+x\right)}{x-y}\right] \\
= & \lim _{b / c \rightarrow 0 ; a / c \rightarrow 0} \frac{\zeta}{Z_{0}} \frac{\bar{V}}{4 \pi \gamma^{\prime}} \frac{b-a}{\sqrt{2} c} \\
& \cdot\left[(x+y) \frac{\delta\left[t^{*}-((x+y) / 2)\right]-\delta\left[t^{*}+((x+y) / 2)\right]}{x+y}\right] \\
= & \frac{\zeta}{Z_{0}} \frac{A}{2 \pi} \frac{\bar{V} \dddot{\delta}\left(t^{*}\right)}{4 \pi r c^{2}} \sin \theta \\
& H_{\phi}(t)=\frac{E_{\theta}(t)}{\zeta} \quad x=\frac{b \sin \theta}{\sqrt{2} c} \quad y=\frac{a \sin \theta}{\sqrt{2} c} \quad(33 \mathrm{~b})
\end{aligned}
$$

thus recovering (26), since $V(t)=\bar{V} \delta(t)$. 


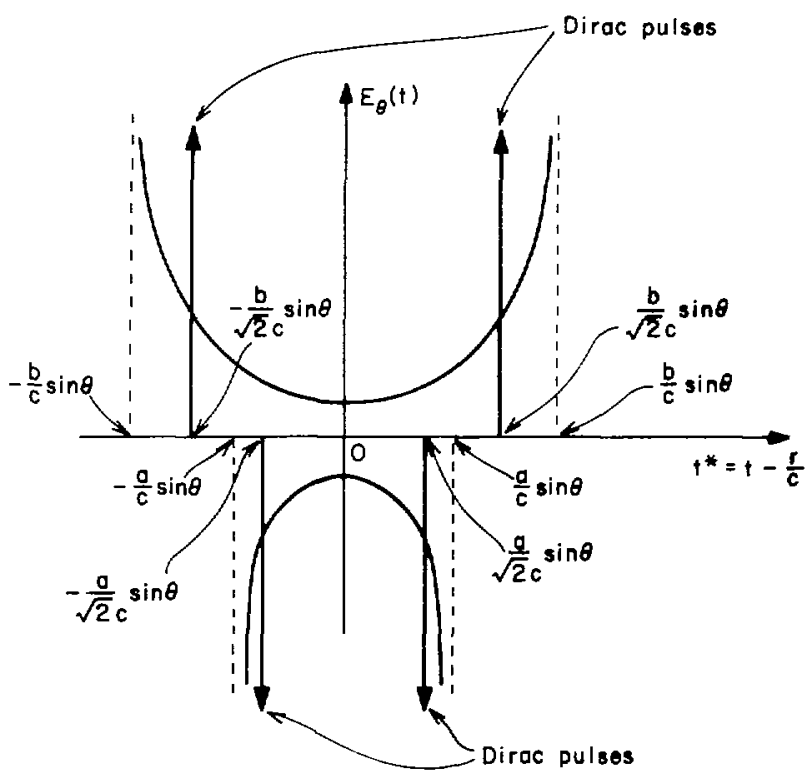

Fig. 5. Plot of field radiated by coaxial circular aperture and transition from continuous radiation to equivalent pulses. Fote that radiated field is superposition of positive and negative plots of figure.

\section{TRANSIENT RADIATION FROAI ANTENNA GAP}

In order to compute the transient radiation from the gap, an infinitely long cylindrical antenna of circular cross section is considered (see Fig. 6). The antenna is excited at a gap of thickness $2 d$ by a step voltage $V(t)=V_{0} U(t)$. Let $E(z, t)=E_{0}(z) U(t) \boldsymbol{e}_{z}$ be the electric field across the gap. The radiated field can be computed by using the equivalence principle [38]. The gap is short-circuited and a magnetic current density

$$
J_{m}(z, t)=E_{0}(z) \delta(\rho-a) U(t) \boldsymbol{e}_{\phi}
$$

is assumed to flow around the antenna, where the gap was located. When the gap thickness is vanishingly small, the magnetic current distribution (34) can be substituted by a ring of magnetic current

$$
I_{m}=\int_{0}^{\infty} d \rho \int_{-d}^{d} J_{m}(z, t) d z=-V_{0} U(t)
$$

On the other hand, the radiation from a loop of electric current was computed in Section III ((21) and (22)). These results can be easily accommodated for the case at hand by using duality (see Section II). For each observation point only one half of the magnetic ring will contribute to the radiation, the other half part being screened by the metallic eylinder. Accordingly, only the single flash point $A$ will be present (see Fig. 6). Furthermore, the radiated field will be doubled, due to the image of the magnetic ring on the metallic conductor.

The resulting radiated field is given by

$$
E_{0}(t)=\frac{V_{0}}{\pi r \sin \theta} \frac{(a / c) \sin \theta-t^{*}}{\left[t^{*}\left((2 a / c) \sin \theta-t^{*}\right)^{-1 / 2}\right.}
$$

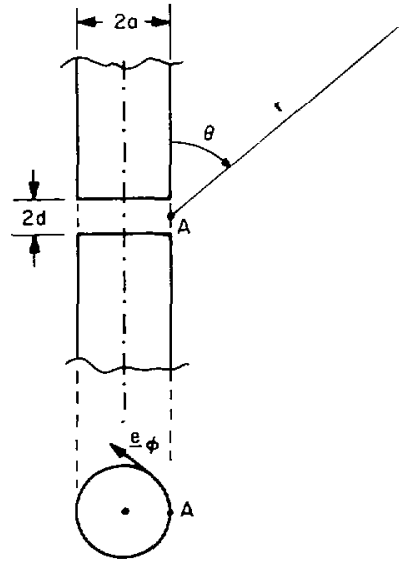

Fig. 6. Relevant to transient radiation from gap of cylindrical antenna.

$$
H_{\phi}(t)=\frac{E_{\theta}(t)}{\zeta}, \quad 0 \leq t^{*} \leq \frac{2 a}{c} \sin \theta
$$

and is zero for $\left.t<0, t^{*}\right\rangle(2 a \sin \theta) / c$. Note that the distances are measured from the flash point $A$.

When $t^{*}=t-r / c$ can be neglected with respect to $(2 a \sin \theta) / c,(36)$ coincide with those describing the earlytime behavior of the radiated field of an infinitely long cylindrical antenna given by Latham and Lee [7]. ${ }^{1}$ When the assumption of infinitesimal gap is relaxed and the gap is allowed to be of finite dimensions, the results given by Pyne and Tesche [32] for the early-time behavior of the field can be obtained.

Equations (36) give the field radiated by the gap, and previous analysis proves that this field coincides with the early-time behavior of the field. Obviously, the launching of the currents onto the antenna will produce other fields, which add to the fields (36). These further terms will be given in Section VI.

The transition from (36)-microscopic approach-to the macroscopic formulation is readily obtained by using the techniques presented in Sections III and IV. The field waveform (36) is substituted by an equivalent pulse, and then $a / c$ is made to approach zero. The field radiated by an applied voltage $V(t)$ can be obtained by differentiating (36) and using convolution. Hence

$$
\begin{aligned}
E_{\theta}(t) & =\frac{\dot{V}\left(t^{*}\right) a}{\pi r c} \\
H_{\phi}(t) & =\frac{E_{\theta}(t)}{\zeta}, \quad t^{*}=t-\frac{r}{c} .
\end{aligned}
$$

\section{ELENENTARY THEORY OF RADIATION FRON LINEAR ANTENNAS AND CIRCLLAR LOOPS}

An approximate analysis of transient radiation from linear antennas and loops of arbitrary dimensions is

\footnotetext{
${ }^{1}$ Note that the voltage applied to the gap is $-V_{0} U(t)$ in [7].
} 
possible by using transmission line techniques for modeling currents and voltages. As far as the cylindrical antenna is concerned, the preceding procedure has already been used [30], [43], [44]. Hereafter it is presented, in a slightly different form appropriate to previous analysis, for completeness and for comparison with experimental results.

A cylindrical antenna of height $2 h$ and diameter $2 a$ is considered (see Fig. 7). If the assumption is made that the antenna is very thin, i.e., $\Omega=2 \ln [2 h / a] \gg 1$, the current distribution in the frequency domain reduces to [39]

$$
\hat{I}(z)=\frac{2 \pi}{\zeta \Omega} V_{\text {in }} \frac{\sin [(\omega / c)(h-\mid z \dot{j})]}{i \cos [\omega h / c]}
$$

where $\hat{V}_{\text {in }}$ is the transformed voltage at the antenna terminals. Equation (38) is formally equivalent to that of the current distribution along an open ended transmission line of length $h$, characteristic impedance $Z_{0}=\zeta \Omega / 2 \pi$ and fed by an ideal voltage generator of zero internal impedance. When the internal impedance of the generator is different from zero and equal to $\alpha Z_{0}$ (real), (38) can be immediately generalized by using the preceding transmission line analogy. Hence

$$
\hat{I}(z)=\frac{\hat{V}}{Z_{0}} \frac{\sin [(\omega / c)(h-|z|)]}{\alpha \sin [(\omega / c) h]+i \cos [(\omega / c) h]}
$$

where $\hat{V}$ is the transformed voltage of the generator.

The transformed fields associated with (39) are readily seen to be

$$
\begin{aligned}
& \hat{E}_{\theta}= \frac{\zeta}{Z_{0}(\alpha+1)} \hat{V} \frac{\exp [i \omega r / c]}{\pi r \sin \theta} \\
& \cdot \frac{\cos [(\omega / c) h]-\cos [(\omega / c) h \cos \theta]}{1+\Gamma \exp [2 i(\omega / c) h]} \\
&= \frac{\zeta}{Z_{0}(1+\alpha)} \hat{V} \frac{\exp [i \omega r / c]}{\pi r \sin \theta} \\
& \cdot\{\cos [\omega h / c]-\cos [(\omega / c) h \cos \theta]\} \\
& \cdot \sum_{0}^{\infty}(-\Gamma)^{n} \exp [2 n i \omega h / c] \\
& \hat{H}=\frac{\hat{E}_{\theta}}{\zeta}
\end{aligned}
$$

where $\Gamma=(1-\alpha) /(1+\alpha)$ is the reflection coefficient.

By Fourier transforming (40) the radiated fields in the time-domain are obtained. For a Dirac pulse excitation $V(t)=V_{0} \delta(t), \hat{V}=V_{0}$ and the radiated fields are given

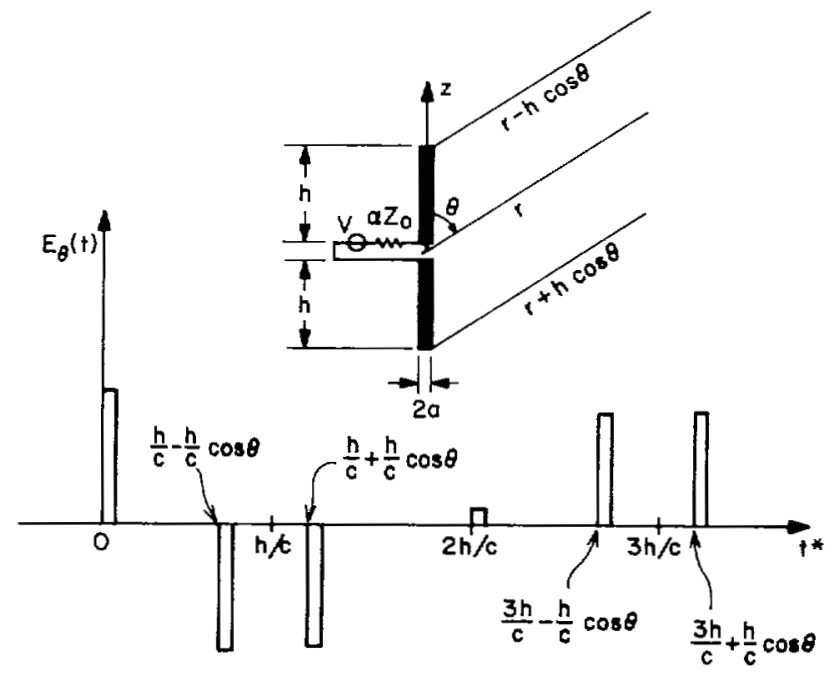

Fig. 7 . Plot of field radiated by linear antenna fed by voltage pulse. Input reflection coefficient $\Gamma$ is assumed to be close to unity.

by

$$
\begin{aligned}
E_{\theta}(t)= & \frac{\zeta}{Z_{0}(\alpha+1)} \frac{V_{0}}{2 \pi r \sin \theta} \\
& \cdot\left\{\delta\left(t^{*}\right)+(1-\Gamma) \sum_{0}^{\infty}(-\Gamma)^{n^{n}}\left(t^{*}-\frac{2(n+1) h}{c}\right)\right. \\
& -\sum_{n}(-\Gamma)^{n}\left[\delta\left(t^{*}-\frac{2 n+1}{c}(h-h \cos \theta)\right)\right. \\
& \left.+\delta\left(t^{*}-\frac{2 n+1}{c}(h+h \cos \theta)\right)\right] \\
& H_{\phi}(t)=\frac{E_{\theta}(t)}{\zeta} .
\end{aligned}
$$

Equations (41) show that the transient radiation of the antenna consists of a chain of Dirac pulses, each being a replica of the applied signal (save for the amplitude and eventually the sign). The first pulse (first term inside the large parenthesis of (41a)) represents the radiation due to the launching of the current along the antenna at time $t=0$. The second series in (41) represents the radiation from the upper and lower tips of the antenna due to the charges associated to the current and going back and forth along the antenna.

The first series in (41) represents the radiation due to the currents relaunched toward the generator. These currents are absorbed in the internal resistance of the generator itself. When the antenna is short-circuited at its terminals $\Gamma=1$, these last terms disappear.

For an arbitrary applied voltage $V(t)$, the radiated field is easily obtained from (41) by using convolution. A sketch 


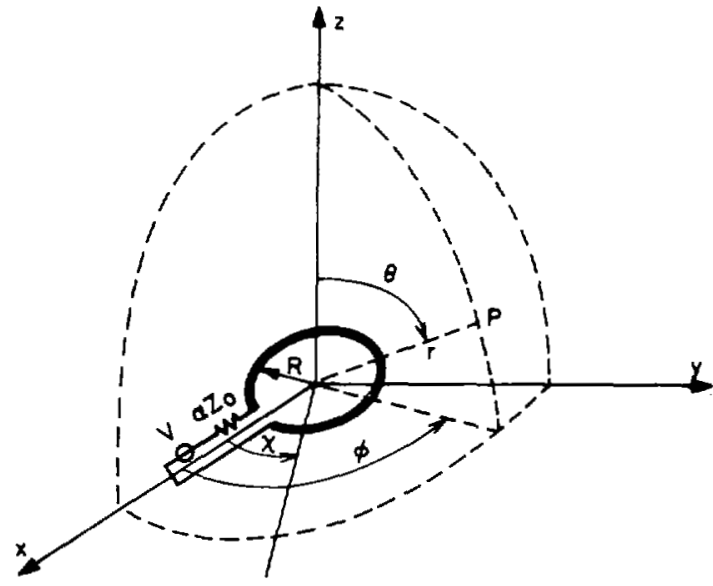

Fig. 8. Relevant to transient radiation from cireular loop.

of the transient field radiated by the antenna when the applied voltage is a rectangular pulse is given in Fig. 7 .

Previous results can be easily applied to the case of a monopole antenna on an infinite perfectly conducting ground plane. The radiation from the lower tip of the antenna disappears, but the transient pulses reflected from the ground plane must be taken into account. In this way, a very satisfactory agreement with the experimental results given in [18] is obtained. ${ }^{2}$

The transient radiation from a circular loop can be obtained following similar lines. The loop of radius $R$ is fed by a voltage generator of internal impedance $\alpha Z_{0}$ (see Fig. 8). The transformed radiative field due to a travelingwave current $I_{0} \exp [i s \chi]$ along the loop is given by [40]

$\widehat{E}_{\phi}=E_{1}\left\{-\frac{J_{1}((\omega / c) R \sin \theta)}{2 s}\right.$

$+\sum_{1}^{\infty} \frac{(-i)^{n} J_{n}{ }^{\prime}((\omega / c) R \sin \theta)}{s^{2}-n^{2}}[s \cos n \phi+i n \sin n \phi]$

$\hat{E}_{\theta}=E_{1} \cos \theta \sum_{1}^{\infty} \frac{(-i)^{n} n J_{n}((\omega / c) R \sin \theta)}{\left(s^{2}-n^{2}\right)(\omega / c) R \sin \theta}$

$\cdot[s \sin n \phi-i n \cos n \phi]$

$\hat{H}=\frac{e_{r} \times \hat{E}}{\zeta}$

where

$$
E_{1}=i \zeta \frac{I_{0} R \omega}{2 \pi r c}(\exp [i 2 \pi s]-1) \exp \left[i \frac{\omega}{c} r\right] .
$$

Expressions (42) are the generalization of the field radiated by a loop with a traveling-wave current $I_{0} \exp [i m \chi], m$ integer. Letting $s=m+\xi$ and then $\xi$ approach zero, the conventional results $[41]$ are recovered.

${ }^{2}$ No checking relative to the amplitude of the fields is, unfortunately, possible due to the lack of quantitative data in [18].
Now, the transformed current along the loop is assumed to be coincident with that of a transmission line of length $\pi R$, characteristic impedance $Z_{0}$ (real) and short-circuited at its end. Hence

$$
\begin{aligned}
\hat{I} & =I_{0} \frac{\cos [(\omega / c) R(\pi-|\chi|)]}{\cos [(\omega / c) R]} \\
& =\frac{\hat{V}}{Z_{0}} \frac{\cos [(\omega / c) R(\pi-\mid \chi \cos [(\omega / c) R]-i \sin [(\omega / c) R]}{\alpha(c) R}
\end{aligned}
$$

where $\hat{V}$ is the transformed voltage of the generator.

Expression (44) can be recast in terms of two travelingwave currents moving in the positive and negative $\chi$ angular coordinate directions. By algebraic manipulation

$$
\hat{I}=\frac{\hat{V}}{Z_{0}(\alpha+1)} \frac{\exp [i(\omega / c) R \chi]+\exp [i(\omega / c) R(2 \pi-\chi)]}{1-\Gamma \exp [2 \pi i(\omega / c) R]} .
$$

From (45) and (42) with $s=\omega R / c$, and using superposition, the transformed field radiated by the loop can be computed. Hence

$$
\begin{aligned}
& \hat{E}=E_{0}\{- \frac{J_{1}((\omega / c) R \sin \theta)}{(\omega / c) R}+2 \frac{\omega}{c} R \\
&\left.\cdot \sum_{1}^{\infty} \frac{(-i)^{n} J_{n}^{\prime}((\omega / c) R \sin \theta)}{(\omega R / c)^{2}-n^{2}} \cos n \phi\right\}
\end{aligned}
$$

$\hat{E}_{\theta}=E_{0} \frac{2 \cos \theta}{\sin \theta} \sum_{2} \frac{(-i)^{n} n J_{n}((\omega / c) R \sin \theta)}{(\omega R / c)^{2}-n^{2}} \sin n \phi$

$\hat{H}=\frac{e_{r} \times \hat{E}}{\zeta}$

where

$$
\begin{aligned}
& E_{0}=i \frac{\zeta V}{Z_{0}(\alpha+1)} \frac{R}{2 \pi r c}\left(\exp \left[i \omega \frac{2 \pi R}{c}\right]-1\right) \\
& \cdot \exp \left[i \frac{\omega}{c} r\right] \sum_{0}^{\infty} \Gamma^{n} \exp \left[2 n \pi i \frac{\omega}{c} R\right] .
\end{aligned}
$$

The solution for the transient radiated field is obtained by Fourier transforming (46). In the case of a Dirac pulse excitation, $V(t)=V_{0} \delta(t)$, and the transform corresponds to the field radiated by a point charge going around in the loop. This transform can be computed in terms of known functions and convolution integrals [45].

\section{CONCLUSIONS AND RECOMMENDATIONS}

In the preceding sections the transient radiation from a number of radiating structures has been considered: elementary electric and magnetic dipoles, coaxial apertures, infinitely long cylindrical antennas, cylindrical antennas, and loops of finite dimensions. It has been shown that two 
approaches-the microscopic and the macroscopic-are possible, depending on the time-scale which is being considered. The procedure for recovering one result from the other has also been exploited.

The cases considered cover most practical applications, and the theory developed seems to be simple, sound, and apt to an engineering development. The only limitation is the neglecting of the dispersion of the pulsed currents along the radiating structures.

In the case of coaxial apertures, this corresponds to neglecting higher order modes of excitation. It is reasonable to surmise that this is acceptable provided that the transverse dimensions of the aperture are small compared to $c T, T$ being the equivalent duration of the applied pulse. This has been rigorously proved by using a frequency domain analysis for the circular coaxial aperture [30].

In the case of cylindrical antennas and loops, the approximation corresponds to neglecting the dispersion of the current pulse injected in the radiating structure. The deformation of the pulses increases as time elapses, so that the deformation becomes more pronounced on "long" radiating structures. Therefore, the analysis presented is acceptable provided that the radiator dimensions are not large compared to $c T$, or, in any case, if reference is made to the early-time behavior of the fields.

A better understanding of the pulse deformation along the radiators is therefore a necessary condition for extending the validity of the results presented in this paper. "A wise man once said that science is no good because it generates ten new problems for every problem it solves. A wiser man answered him by saying that science is good because it uncovers ten new problems for every problem it solves" [42].

\section{ACKNOWLEDGMENT}

The authors are indebted to R. Stratton for the careful typing of the manuscript.

\section{REFERENCES}

[1] T. T. Wu, "Introduction to linear antennas," in Antenna Theory, R. E. Collin and F. J. Zucker, Eds. New York: MeGraw-Hill, 1969, vol. 1, eh. 8, p. 350.

[2] P. O. Brundell, "Transient electromagnetic waves around a cylindrical transmitting antenna," Erricsson Tech., vol. 16 no. 1 , pp. $137-162,1960$.

[3] T. T. Wu, "Transient response of a dipole antenna," J. Math. Phys., vol. 2, no. 6, pp. 892-894, 1961.

[4] S P. Morgan, "Transient response of a dipole antenna," $J$. Math. Phys., vol. 3, no. 3, pp. 564-565, 1962.

[5] O. Einarsson, "The step voltage current response of an infinite conducting cylinder," Trans. Roy. Inst. Technol. Stockholm, vol. 191,1962 .

[6] C. W. Harrison and R. W. P. King, "On the transient response of an infinite cylindrical antenna," IEEE Trans. Antennas Propagat. (Commun.), vol. AP-15, pp. 301-302, Mar. 1967.

[7] R. W. Latham and K. S. H. Lee, "Transient properties of an infinite cylindrical antenna," Radio Sci., vol. 5, no. 4. pp. 715723,1970 .

[8] T. T. Wu and R. W. P. King, "Transient response of linear antennas driven by a coaxial line," IEEE Trans. Antennas Propagat., vol. AP-11, pp. 17-23, Jan, 1963.

[9] R. J. Palciauskas and R. E. Beam, "Transient fields of thin cylindrical antennas," IEEE Trans. Antennas Propagat. (Commun.), vol. AP-18, pp. 276-278, Mar. 1970.

[10] A. M. Abo-Zena and R. E. Beam, "Electromagnetic fields at points near pulse-excited linear antenna," IEEE Trans. $A n$ - tennas Propagat. (Commun.), vol. AP-19, pp. 129-131, Jan. 1971.

[11] C. W. Harrison and C. S. Williams, "Transients in wide angle conical antennas," IEEE Trans. Antennas Propagat., vol. AP-13, pp. 236-246, Mar. 1965.

[12] C. Polk, "Transient behavior of aperture antennas," Proc. IRE, vol. 48, pp. 1281-1288, July 1960 .

[13] D. K. Cheng and F. I. Tseng, "Transient and steady-state antenna pattern characteristics for arbitrary time signals," IEEE Trans. Antennas Propagat. (Commun.), vol. AP-12, pp. $492-493$, July 1964.

[14] D. C. Chang and C. W. Harrison, "On the pulse response of a flush-mounted coaxial aperture," IEEE Trans. Electromagn. Compat., vol. EMC-13, pp. 14-18, Feb. 1971.

[15] A. M. Abo-Zena and R. E. Beam, "Transient radiation field of a circular loop antenna," IEEE Trans. Antennas Propagat. (Commun.), vol. AP-20, pp. 380-383, May 1972.

[16] A. R. Dion, "Transmission of step functions by loop antennas," IEEE Trans. Antennas Propagat. (Commun.), vol. AP-18, pp. 389-392, May 1970.

[17] D. L. Sengupta and Y. P. Liu, "Waveforms radiated by continuously loaded linear antennas excited by Gaussian pulse," presented at Spring URSI Meeting, Washington, D. C., 1972.

[18] H. J. Schmitt, C. W. Harrison, and C. S. Williams, Jr., "Calculated and experimental response of thin cylindrical antennas to pulse excitation," IEEE Trans. Antennas Propagat., vol. AP-14, pp. 120-127, Mar. 1966.

[19] D. Lamensdorf, "The transient response of the coaxial cone antenna," IEEE Trans. Antennas Propagat. (Commun.), vol. AP-18, pp. 799-802, Nov. 1970 .

[20] R. W. P. King and H. J. Schmitt, "The transient response of linear antennas and loops," IRE Trans. Antennas Propagat., vol. AP-10, pp. 222-228, May 1962.

[21] H. J. Sehmitt, "Experimental observation of the transient response of linear antennas and loops," IEEE Trans. Antennas Propagat. (Commun.), vol. AP-11, pp. 509-510, July 1963.

[22] C. E. Baum, "Electromagnetic transient interaction with objects with emphasis on finite size objects, and some aspects of transient pulse production," presented at Spring URSI Meeting, Washington, D. C., 1972.

[23] F. M. Tesche, "On the analysis of seattering and antenna problems using the singularity expansion technique," IEEE Trans. Aniennas Propagat., vol. AP-21, pp. 53-62, Jan. 1973.

[24] C. L. Bennett and J. Martine, "A space-time integral equation solution for currents on wire structures with arbitrary exeitation," presented at Fall URSI Meeting, Columbus, Ohio, 1970.

[25] A. J. Poggio "The space-time domain magnetic vector potential integral equation," IEEE Trans. Antennas Propagat. (Commun.), vol. AP-19, pp. 702-704, Sept. 1971.

[26] C. L. Bennett and A. M. Auckenthaler, "Transient and timedomain solutions for antennas and scatterers," IEEE Int. Conv. Dig., pp. 624-625, 1971.

[27] E. P. Sayre and R. F. Harrington, "Time-domain radiation and scattering by thin wires," Appl. Sci. Res., vol. 26, no. 6, pp. $413-444,1972$.

[28] E. K. Miller, A. J. Poggio, and G. J. Burke, "An integrodifferential equation technique for the time-domain analysis of thin wire structure, 1 , the numerical method," J. Comput. Phys., vol. 12 , no. 1 , pp. $24-48,1973$.

[29] T. K. Lui and K. K. Mei, "A time-domain integral equation solution for linear antennas and scatterers," Radio Sci., vol. 8 , nos. 8-9, pp. 797-804, 1973.

[30] N. L. Broome, "Transient radiation from coaxial waveguide and cylindrical monopole antennas," Caltech Antenna Lab., Pasadena, Calif., Rep. 67, 1973.

[31] F. M. Tesche, "The effect of the thin wire approximation and the source gap model on the high-frequency integral equation solution of radiating antennas," IEEE Trans. Antennas Propagat. (Commun.), vol. AP-20, pp. 210-211, Mar. 1972.

[32] Z. L. Pyne and F. M. Tesche, "Calculation of the early time radiated eleetric field from linear antenna with a finite source gap," IEEE Trans. Antennas Propagat., vol. AP-21, pp. 740743,1973

[33] C. H. Papas, "Pulsed antennas," in Yerevan Lectures on Electromagnetic Theory, pp. 64-71, 1972.

[34] P. I. Richards, "Transients in conducting media," IRE Trans. Antennas Propagat., vol. AP-6, pp. 178-182, Apr. 1958.

[35] J. R. Wait, "Propagation of electromagnetic pulses in a homogeneous conducting earth," A ppl. Sci. Res. Sect. B, vol. 8, no. 3 , pp. 213-253, 1960.

[36] - , "Exact field of pulsed dipole in a homogeneous cold plasma," J. A ppl. Phys., vol. 41, no. 7, 2880-2884, 1970.

[37] J. R. Wait and D. A. Hill, "Transient signals from a buried magnetic dipole," $J$. Appl.' Phys., vol. 42, no. 10, pp. 3866$3869,1971$.

[38] R. F. Harrington, Time-Harmonic Electromagnetic Fields. New York: McGraw-Hill, 1961, ch. 3, pp. 110-113. 
[39] J. D. Kraus, Antennas. New York: MeGraw-Hill, 1950, ch. 9, pp. 235-247 and 247-249.

[40] S. M. Prasad and B. N. Das, "A circular loop antenna with traveling-wave current distribution," IEEE Trans. Antennas Propagat. (Commun.), vol. AP-18, no. 2, pp. 278-280, Mar. 1970.

[41] R. W. P. King, "The loop antenna for transmission and reception," in Antenna Theory, R. E. Collin and F. J. Zucker, Eds. New York: MeGraw-Hill, 1969, vol. 1, ch. 11, pp. 458-482.

[42] C. H. Papas, "Pulsed antennas," in Yerevan Lectures on Electromagnetic Theory, p. 79, 1970.

[43] V. C. Martins, "A method for sketching the transient electromagnetic response radiating from a dipole-monopole antenna excited by a general source impedance and driving function," in 1968 Int. Symp. on Antennas and Propagation, Boston, Mass.

[44] B. N. Krasil'nikov and G. I. Makarov, "Nonstationary Processes in Linear Oscillators," Vestnik Leningradskogo Cniversiteta, $16,27-50,1958$

[45] G. Franceschetti and C. H. Papas, "Pulsed antennas," Caltech Antenna Lab., Pasadena, Calif., Rep. 70, 1973.

\section{Additional References}

[16] K. Franz, "Behavior of dipole impedance with time exponential excitation of dipole," Arch. Elek. Übertragung, vol. 14, no. 4, pp. 167-168, 1960.

[47] H. J. Frankena, "Transient phenomena associated with Sommerfield's horizontal dipole problem," Appl. Sci. Res. Sec. B, vol. 8, no. 4, pp. $357-358,1960$.

[48] A. T. de Hoop and H. J. Frankena, "Radiation of pulse generated by a vertical dipole above a plane nonconducting earth," $A$ ppl. Sci. Res. Sec. B, vol. 8, no. 4, pp. 369-377, 1960.

[49] D. M. Bolle, "The radiation pattern of long thin antennas for short-pulse excitation," IRE Trans. Antennas Propagat., vol. AP-10, pp. 787-788, Sept. 1962.

[50] C. R. Burrows, "Transient response in an imperfect dielectric," IEEE Trans. Antennas Propagat., vol. AP-11, pp. 286-296, May 1963.

[51] C. W. Harrison, "Physical limitations on the measurement of transient fields in air and dissipative media using electric and magnetic probes," IEEE Trans. Antennas Propagat., vol. AP-12, pp. 530-533, Sept. 1964.

[52] E. A. Lewis, J. E. Rasmussen and J. R. Stahmann, "Waveforms and relative phase stability of transients radiated from a helicopter-supported antenna wire," IEEE Trans. Antennas Propagat., vol. AP-13, pp. 257-261, Mar. 1965.

[53] D. E. Mereucther, "Transient pulse transmission using impedance bonded cylindrical antennas," Ph.D. dissertation, Univ. Microfilms (from University of New Mexico, Albuquerque) order No. 68-13077, 1968.

[54] E. P. Sauree and R. F. Harrington, "Transient response of straight wire scatterers and antennas," in 1968 Int. Symp. on Antennas and Propagation. Boston, Mass., pp. 160-164.

[55] C. L. Bennett and W. H. Weeks, "Electromagnetic pulse re sponse of cylindrical seatterers," in 1968 Int. Symp. on Antennas and Propagation, Boston, Mass., pp. 176-183.

[56] R. H. Ott, "The time domain backscatter from dipole antennas illuminated at broadside," Air Force Cambridge Research Labs., Rep. 68-0808, 1968.

[57] R. J. Palciauskas, "Transient fields of thin eylindrical antennas," Ph.D. dissertation, Univ. Microfilms (from Northwestern, Evanston, Ill.) order no. 69-2906, 1968.

[58] R. H. Ott, "The time domain backscatter from dipole antennas illuminated at broadside," Sci. Rep. B-2 Rev., (AD688209), 1968.

[59] C. D. Taylor and T. H. Shumpert, "Electromagnetic pulse generation by an impedance loaded dipole antenna," IEEE Trans. Antennas Propagat. (Commun.), vol. AP-18, pp. 110112, Jan. 1970.

[60] J. R. Wait and K. P. Spies, "Transient magnetic field of a pulsed electric dipole in a dissipative medium," IEEE Trans. Antennas Propagat. (Commun.), vol. AP-18, pp. 714-716, Sept, 1970.

[61] C. M. Knop, "On transient radiation from a log-periodic dipole array," IEEE Trans. Antennas Propagat. (Commun.), vol. AP-18, pp. 807-808, Nov. 1970.

[62] A. M. Abo-Zena and R. E. Beam, "Transient radiation field of a pulse excited linear antenna parallel to a conducting plane," in 1970 Int. Symp. on Antennas and Propagation, Columbus, Ohio, pp. 310-317.

[63] - - "Electromagnetic fields at points near pulse-excited linear antenna," IEEE Trans. Antennas Propagat. (Commun.), vol. AP-19, pp. 129-131, Jan. 1971.

[64] Chen-to Tai and H. E. Foster, "Processes of transient radiation and reception," in 1971 Inter. Symp. on Antennas and Propagation, Sundai, Japan, pp. 1-2.

[65] A. M. Abo-Zena and B. E. Beam, "Transient radiation fields of a pulse-excited loop antenna parallel to a conducting plane," in 1971 Int. Symp. on Antennas and Propagation, Sundai, Japan, pp. 3-4.

[66] D. L. Waidelick, "Response of pulsed dipoles in conductors," in 1971 Int. Symp. on Antennas and Propagation, Sundai, Japan, pp. 5-6.

[67] Y. K. Lin and K. K. Mei, "A line domain integral equation solution for thin wire dipoles," in 1971 Int. Symp. on Antennas and Propagaiton, Sundai, Japan, pp. 13-14.

[68] S. F. Fralick, "Radiation of electromagnetics waves with Walch-function time variation. Preliminary results," in 1972 IEEE Int. Conf. on Communications, Philadelphia, $\mathrm{Pa}$., pp. 38-164.

[69] V. P. Pyati, R. W. Graff, and L. J. Ricardi, "Comments on a transform solution for the fields from a current element, Trans. Antennas Propagat. (Commun.), vol. AP-21, p. 132, Jan. 1973. 